\title{
El Turismo como una estrategia para el mundo en desarrollo: el Programa UNWTO. Volunteers
}

\author{
Susana Lima ${ }^{i}$ \\ Instituto Politécnico de Coimbra - ESEC (Portugal) \\ Cecilia S. García Gómez ii \\ Fundación UNWTO.Themis (México) \\ Domingo Gómez López iii \\ Universidad Intercultural de Chiapas (México) \\ Celeste Eusébio iv \\ Universidad de Aveiro - DEGEI (Portugal)
}

\begin{abstract}
Resumen: El compromiso del turismo como una estrategia para el mundo en desarrollo vine adquiriendo mayor interés de los gobiernos y de las organizaciones internacionales para el cumplimiento de los Objetivos de Desarrollo del Milenio. En este artículo, se busca contribuir al marco teórico relacionado con las diversas perspectivas sobre los programas de cooperación internacional para el desarrollo, que consideran al turismo como una estrategia que contribuye al logro de esos objetivos, como es el caso del Programa UNWTO.Volunteers. Las características principales de este programa serán descritas y se discutirán los impactos que pueden producir en los destinos involucrados. Esta discusión se basa en un estudio de caso del Programa UNWTO.Volunteers, desarrollado en el estado mexicano de Chiapas en 2008.
\end{abstract}

Palabras clave: Turismo; Pobreza; Gestión del conocimiento; Cooperación internacional para el desarrollo; Programa UNWTO.Volunteers; Chiapas.

Title: Tourism as a strategy for the developing world: Agenda UNWTO.Volunteers

Abstract: In recent years, commitment to tourism as a development strategy for the developing world has gained an increased interest from the governments and development organizations in the fulfilment of the United Nations Millennium Development Goals. This paper looks to contribute to the theoretical discussion related to the diverse perspectives about the role of international development cooperation programs that use tourism as a main tool for development, as is the case of UNWTO.Volunteers Program. The main characteristics of this program are described and its potential results are discussed considering the impacts it may produce on the destination areas involved. This discussion is based upon an UNWTO.Volunteers Program case study, developed in the Mexican state of Chiapas in 2008.

Keywords: Tourism; Poverty; Knowledge management; International development assistance; UNWTO.Volunteers Program; Chiapas.

\footnotetext{
i Profesora de la licenciatura en Turismo de la Escola Superior de Educação de Coimbra (ESEC), doctoranda en Turismo e investigadora de la Unidad de Investigación en Gobernanza, Competitividad y Políticas Públicas (GOVCOPP) de la Universidad de Aveiro, Portugal. Email:sulima@esec.pt.

ii Colaboradora y Experta de la Fundación UNWTO.Themis, México. Email: cgarciadecortes@yahoo.com.mx.

iii Profesor de Tiempo Completo de la Licenciatura en Turismo Alternativo de la Universidad Intercultural de Chiapas, México. Director General del Centro de Investigación para el Desarrollo Sustentable (CIDES). Email: domingomezl@gmail.com.

iv Profesora Auxiliar en Turismo en el Departamento de Economia, Gestão e Engenharia Industrial (DEGEI), GOVCOPP, de la Universidad de Aveiro, Portugal. Email: celeste.eusebio@ua.pt.
} 


\section{Introducción}

El turismo presenta un enorme potencial para la atenuación de la pobreza en los países en desarrollo, que es el objetivo aglutinador de los ocho Objetivos de Desarrollo del Milenio (ODM) de las Naciones Unidas. Para lograrlo se puede apelar a diversos tipos de intervenciones, por ejemplo, el soporte de programas de cooperación internacional para el desarrollo, con la capacitación de las comunidades locales de los destinos objeto de intervención, a través de una efectiva transferencia de conocimiento, para que puedan perpetuar un desarrollo sostenible, basado en una sólida gobernanza, implicando el sector público, el sector privado y la sociedad civil.

En los últimos años, se verificó un interés creciente por el turismo como un instrumento de atenuación de la pobreza y el hambre, por parte de los gobiernos, de los donadores, de instituciones bilaterales y multilaterales, organizaciones no gubernamentales, organismos oficiales nacionales e internacionales de turismo. En este ámbito, destaca el papel de la Organización Mundial del Turismo, que asumió el compromiso con los ODM como una prioridad. El Programa UNWTO.Volunteers es un ejemplo de ello.

El diseño e implementación del Programa presupone precisamente la transferencia de conocimiento para los destinos de los países en desarrollo, en proyectos que involucran todos los actores clave para el desarrollo sostenible del turismo, del sector público, sector privado y sociedad civil, buscando la excelencia de la gobernanza, y por esta vía contribuir para la atenuación de la pobreza en los países en desarrollo.

Es con este enfoque que se pretende discutir en este artículo el papel del turismo como instrumento de desarrollo, a través de programas de cooperación internacional, tomando como ejemplo el Programa UNWTO.Volunteers y el estudio de caso de su implementación en el estado mexicano de Chiapas, en 2008. En una primera parte, discutimos el potencial aporte del turismo para alcanzar los Objetivos de Desarrollo del Milenio, considerando la complejidad del concepto de pobreza, siguiéndose un breve abordaje de los tipos de programas de ayuda internacional al desarrollo. Se presentan las características principales del Programa UNWTO.Volunteers, terminando con el caso del Proyecto "Chiapas 2015", donde se presentan sus principales fases y se discuten sus potenciales impactos.

\section{El papel del turismo para lograr los Objetivos de Desarrollo del Milenio}

El turismo es uno de los mayores motores económicos en el mundo contemporáneo y puede tener un papel primordial en la realización de los objetivos establecidos por las Naciones Unidas, en la Declaración del Milenio de 2000 , la cual constituye una visión colectiva de la cooperación global para el desarrollo en el siglo XXI.

Después de varias conferencias globales en la década de 1990, en las cuales se fueron definiendo los objetivos de desarrollo para el milenio, fue en aquella ocasión que los 189 Estados Miembros de las Naciones Unidas establecieron objetivos medibles en todas las esferas de actividad consideradas determinantes para el desarrollo de la humanidad, teniendo como meta el año 2015. Esta visión de futuro asumió así la forma de ocho ODM, que se describen a continuación (UN, 2009):

Objetivo 1: Erradicar la pobreza extrema y el hambre

Objetivo 2: Lograr la enseñanza primaria universal

Objetivo 3: Promover la igualdad entre los sexos y el empoderamiento de la mujer

Objetivo 4: Reducir la mortalidad de los niños menores de 5 años

Objetivo 5: Mejorar la salud materna

Objetivo 6: Combatir el VIH/SIDA, la malaria y otras enfermedades

Objetivo 7: Garantizar la sostenibilidad del medio ambiente

Objetivo 8: Fomentar una alianza mundial para el desarrollo

Entre los ODM se destaca el primero por considerarse que de su concretización dependerá la de los restantes objetivos. La ausencia de las condiciones mínimas para vivir dignamente constituye así el eje central de la actuación del combate centrado en la lucha contra la pobreza, que se plasma en uno de los mayores desafíos en el marco de la cooperación para el desarrollo sostenible, en un mundo cada vez más globalizado e interdependiente, donde la reducción de las desigualdades entre países desarrollados y en desarrollo se asume no solamente como un imperativo ético y moral, sino como una prioridad en términos de seguridad global.

Por consiguiente, el turismo se asume como una actividad de excelencia para combatir la extrema pobreza, pudiendo constituirse como un importante instrumento de desarrollo de regiones desfavorecidas, si es conducido debidamente y teniendo como marco de acción, los principios elementares del desarrollo sostenible.

En esta perspectiva, es fundamental ante todo comprender el complejo concepto de pobreza, en sus distintas dimensiones, ya que no se trata solamente de bajos rendimientos, sino también de aspectos como la salud y educación, privación del conocimiento y comunicación, inhabilidad para ejercer los derechos humanos y políticos y la ausencia de dignidad, confianza y auto-expresión (UNDP, 1997). Asimismo, el Programa de las Naciones Unidas para el Desarrollo (PNUD) definió tres perspectivas de la pobreza: la perspectiva del rendimiento, la perspectiva de las necesidades básicas y la perspectiva de la capacidad. En este contexto, es posible definir la pobreza utilizando el abordaje económico, basado esencialmente en el rendi- 
miento y consumo - medido por el Banco Mundial a través del PIB per capita - y no-económico, que incorpora tres elementos esenciales de la vida humana como la longevidad, conocimiento y un nivel de vida decente - medido por el Índice de Pobreza Humana (IPH), que fue introducido por el PNUD en 1997.

Asimismo, actualmente, existen más de 125 naciones en desarrollo, si consideramos el bajo rendimiento per cápita y el mediano o bajo nivel de desarrollo humano, según los criterios mencionados arriba (UN, 2006).

En el actual escenario, ¿cómo puede entonces el turismo contribuir para atenuar la pobreza en sus diversas dimensiones (Lima et al., 2010)?

El turismo se ha convertido en un motor clave del progreso socioeconómico, con un crecimiento sostenido y una diversificación cada vez mayor, en los últimos seis decenios. Si bien en 1950, los quince primeros destinos turísticos representaron el 88\% de las llegadas internacionales, en 2007 el porcentaje cayó al $57 \%$, lo que refleja el surgimiento de nuevos destinos, muchos de ellos en países en desarrollo (UNWTO, 2008).

Según la UNWTO (2008), en la actualidad, los ingresos de exportación generados por el turismo internacional ocupan la cuarta posición, después de los combustibles, los productos químicos y los productos automotrices, mientras que en muchos países en desarrollo el turismo es la primera categoría de exportación representando una de las principales fuentes de ingresos para muchos países en desarrollo, crea empleo y oportunidades de desarrollo. De hecho, las llegadas de turistas en todo el mundo ascendieron a 903 millones en 2007, lo que representa un crecimiento anual del 6,6 \%; entre 1995 y 2007 el crecimiento se situó en promedio arriba del $4 \%$ al año; los ingresos por turismo internacional se elevaron a 625000 millones de euros, en 2007, lo que corresponde a un incremento en términos reales del 5,6\% en relación con 2006. No obstante, el año 2008 presentó un crecimiento más moderado de $2 \%$ (924 millones de llegadas), debido a la crisis de la economía global, que afectó la confianza del consumidor, el aumento del paro y la reducción del ingreso disponible. Aún así, según las previsiones de la Organización Mundial de Turismo (OMT - UNWTO) para 2020, el número de llegadas mundiales será de 1600 millones (UNWTO, 2001; UNWTO, 2008).

Estos datos y cifras demuestran que el turismo juega un papel muy importante para la generación de ingresos y creación de empleo. Sin embargo, si la importancia económica del turismo para los países en desarrollo ya es ampliamente reconocida, su rol como instrumento de lucha contra la pobreza es muy reciente (Zhao \& Ritchie, 2007).

En UNWTO (2004) se identifican siete diferentes formas en que el turismo puede funcionar como instrumento de reducción de la pobreza: (1) empleo de los pobres en empresas turísticas; (2) oferta de bienes y servicios a las empresas turísticas por los pobres o empresas que emplean pobres; (3) ventas directas de bienes y servicios por los pobres a los visitantes; (4) creación de empresas turísticas por los pobres; (5) tasas o impuestos sobre el rendimiento turístico que van a beneficiar los pobres; (6) donativos a los pobres por los turistas o empresas turísticas; (7) inversión en infraestructura estimulada por el turismo y que también beneficia a los pobres.

El problema es que, no obstante el crecimiento que el turismo pueda generar en los países en desarrollo, hay potenciales contradicciones que lo impiden, tales como: falta de recursos humanos calificados para trabajar en el sector; elevado control extranjero sobre los recursos locales; sustancial fuga de capitales al exterior; falta de articulación con otros sectores de la economía; bajo efecto multiplicador fuera de los enclaves turísticos; refuerzo de los padrones de desigualdad socioeconómica y espacial (Brohman, 1996; Carbone, 2007; Scheyvens \& Momsen, 2008).

Según Zhao \& Ritchie (2007), esto ocurre porque la reducción de la pobreza ha sido considerada como un aporte natural del crecimiento económico regional que el turismo puede inducir y no como una prioridad. Sin embargo, en los últimos años, se verificó un interés creciente por el turismo como un instrumento de atenuación de la pobreza y del hambre, por parte de los gobiernos, de los donadores, de instituciones bilaterales y multilaterales, organizaciones no gubernamentales (ONG), organismos oficiales nacionales e internaciones de turismo. En este ámbito, destaca el papel de la Organización Mundial del Turismo, que asumió el compromiso con los ODM como una prioridad, como demuestra su imagen institucional en la página Web (UNWTO, 2009) en la cual destaca el lema "UNWTO - Comprometidos con el turismo y con los objetivos de desarrollo del Milenio".

\section{El turismo y la cooperación internacional para el desarrollo}

Hay varias formas de abordar el tema de la asistencia al Desarrollo internacional en el cual el Turismo se considera la principal herramienta para la mitigación de la pobreza. El abanico de programas y tipos de instituciones que se pueden considerar en este debate es muy variado, considerando desde distintas Organizaciones no Gubernamentales, agencias de ayuda internacional, el Banco Mundial, la Organización Mundial del Turismo a la Comisión Europea entre otras que trabajan sin excepción, aunque con diferentes abordajes, para el mismo objetivo que es la mitigación de la pobreza.

Considerando el vasto conjunto de iniciativas y programas existentes actualmente hay una necesidad de entender las diferentes naturalezas, tipos de intervención y resultados, lo que todavía no se refleja en la literatura científica sobre turismo y desarrollo. El presente artículo pretende aportar conocimiento en este dominio, incidiendo sobre la importancia del principio de la transferencia de conocimiento y experiencia desde los países desarrollados, en vez de enfocarse a intervenciones esporádicas en varios proyectos desarticulados entre sí. Esto ocurre a menudo y no contribuye para una eficaz "construcción 
de capacidades" (traducción literal del término capacitybuilding) a largo plazo en los destinos de los países en desarrollo (Lima et al., 2010).

La "construcción de capacidades" es un concepto fundado en los principios básicos de la democracia, participación, desarrollo, y mejoría continua de los conocimientos así como los procedimientos de aprendizaje compartidos y el acceso igualitario a las oportunidades. El reaparecimiento del debate sobre el desarrollo sostenible antes y después de la Conferencia de las Naciones Unidas sobre el Medio Ambiente y Desarrollo, en 1992, ha contribuido para colocar la "construcción de capacidades" en el primer lugar de la lista de prioridades de la asistencia al desarrollo internacional y como condición necesaria para permitir la realización efectiva y el desarrollo profundo de todas las regiones del planeta (Partidário \& Wilson, 2009).

La conciencia de que todas las personas y comunidades, incluso las afectadas por un desastre, disponen de determinadas capacidades propias ha reforzado una visión de los receptores de la ayuda internacional no como "víctimas" pasivas, sino como agentes activos de su propio desarrollo, cuya participación es siempre necesaria. Consiguientemente, la denominada construcción de capacidades ha emergido como uno de los principales cometidos que debe perseguir dicha ayuda. Ésta se suele entender en dos sentidos, que resultan complementarios: a) el primero, como la creación de capacidades de las personas mediante su formación, concienciación y organización, de forma que puedan articular sus intereses y promover el cambio social, en otras palabras, como un proceso de empoderamiento; b) el segundo, como un desarrollo institucional, esto es, la creación y refuerzo de organizaciones o instituciones locales que enriquezcan la sociedad civil y defiendan los intereses de los pobres, a lo cual se puede contribuir, por ejemplo, mediante la formación de recursos humanos en gestión y planificación, o el establecimiento de redes institucionales (Dubois, 2007) .

Actualmente, se reconoce que la construcción de capacidades no trata solamente sobre la asistencia al desarrollo pero que también implica la implementación técnica y la toma de decisiones en todos los países y regiones que conllevará a nuevos caminos que permitirán que el medio ambiente y los asuntos sociales sean una parte integral del desarrollo económico (Partidário \& Wilson, 2009). Hay que agregar además que el turismo tiene impactos que rebasan el ámbito económico. Más allá del desplazamiento de personas, de un lugar habitual de permanencia a otro temporal, que implica transporte, alojamiento, alimentación y entretenimiento, el turismo es un fenómeno social complejo, con múltiples facetas que involucran la esfera de lo económico, lo político, lo social, lo cultural y lo ambiental (Fayos-Solà, 2004; Fayos-Solà \& Pedro, 2001).

El turismo ha sido apoyado cada vez más por las agencias multilaterales y bilaterales de desarrollo como una estrategia de reducción de la pobreza y de concretización de los ODM.

\section{Instituciones Multilaterales}

Hay una creciente demanda para usar el turismo como una herramienta de desarrollo que se refleja en la existencia de un vasto conjunto de proyectos y programas apoyados por instituciones especializadas que además facilitan asesoría técnica en los países en desarrollo. De hecho, estas instituciones multilaterales incluyen los bancos Africano, Asiático e Interamericano de Desarrollo, la Unión Europea y diversas agencias de las Naciones Unidas como la Organización Mundial de Turismo, el Programa de las Naciones Unidas para el Desarrollo, el Programa de Educación de las Naciones Unidas, la Organización de las Naciones Unidas para la Educación, la Ciencia y la Cultura y, la Conferencia de Comercio y Desarrollo de las Naciones Unidas que están todas envueltas en actividades relacionadas con el turismo desde una variedad de perspectivas (Hawkins \& Mann, 2007).

\section{Instituciones Bilaterales}

Aparte de las instituciones internacionales a las que se ha mencionado, las agencias de desarrollo con delegaciones en el extranjero están financiando cada vez más proyectos de desarrollo con base en el turismo y son demasiadas para ser enumeradas en este artículo. Algunos ejemplos son las agencias Alemana, Holandesa y Española, todas con el objetivo específico de postular al turismo como herramienta para el desarrollo y la reducción de pobreza (Ferguson, 2007; Hawkins \& Mann, 2007).

Existe además una variedad de Organizaciones No Gubernamentales que tienen objetivos específicos para el turismo particularmente en lo que se refiere al desarrollo de las comunidades y la conservación.

\section{Determinantes para la eficacia del turismo como instrumento de desarrollo}

Para que el turismo se pueda constituir como una verdadera herramienta de desarrollo a través de los diferentes proyectos y programas como en el caso de los ejemplos anteriormente mencionados, la asesoría técnica por ellos realizada deberá considerar algunos aspectos considerados determinantes para lograr su eficacia. A continuación se identifican esos determinantes.

La participación comunitaria es esencial al desarrollo ya que produce decisiones más apropiadas (Cole, 2006). El turismo amplía el espectro de participación, comparado con otros sectores no agrícolas y emplea un elevado porcentaje de mujeres (Chok et al. 2007). Siendo una industria de servicios, este sector es altamente dependiente de la buena disposición y cooperación de las comunidades receptoras. La medida en que los pobres pueden beneficiarse del turismo depende de la forma en que se involucran con la actividad (Blake et al. 2008). Tosun y Timothy (2003) argumentan además que la comunidad local tendrá más posibilidades de saber qué puede funcionar y qué no funcionará en las condiciones locales. No obstante, existen límites operativos, estructurales y culturales a la participación comunitaria en los procesos de desarrollo turístico 
de muchos países en desarrollo (Tosun, 2001). Además, algunas iniciativas de turismo para combatir la pobreza en estos países pueden contribuir a la manipulación de oportunidades para servir a los intereses de los actores más poderosos, produciendo un desarrollo desigual (Brohmam 1996; Chok et al. 2007) si no se toman las medidas adecuadas. Uno de los principales desafíos de los programas de ayuda para el desarrollo es superar estas barreras.

Otra determinante que debe ser considerada es el empoderamiento (empowerment). Aunque este es un término utilizado con frecuencia en los trabajos sobre desarrollo, no existe un consenso respecto a su definición. Eade (1997, p.4) lo define como el proceso de "ganar la fortaleza, confianza y visión para trabajar por cambios positivos en las vidas [de mujeres, hombres y niños] individualmente y junto con otros". Agrega además que "hombres y mujeres son empoderados por sus propios esfuerzos, no por lo que otros hacen por ellos" y de ahí que "cuando los programas de desarrollo no están fundamentados firmemente en los esfuerzos de las personas por cambiar, su impacto puede ser totalmente contrario" (disempowering). Cole (2006, p.631) sostiene que el estadio final de la pirámide de participación se presenta cuando los miembros de una comunidad son agentes de cambio activos y cuando ellos tienen la capacidad de encontrar soluciones a sus problemas, tomar decisiones, llevar a cabo acciones y evaluar sus soluciones. En especial, el concepto de empoderamiento es enfatizado por Scheyvens (1999) como la importancia de que las comunidades locales tengan un elevado grado de control sobre las actividades turísticas que tienen lugar en sus áreas, compartiendo sus beneficios. Para que esto ocurra, con frecuencia es necesario que los estados intervengan para proporcionar legislación y apoyos en términos de información y adiestramiento (Scheyvens, 2007).

Por otra parte, aún cuando el conocimiento es un elemento crucial del empoderamiento (Tosun, 2001), las comunidades necesitan tener acceso a un amplia gama de información. Más aún, "no puede darse una participación significativa antes de que una comunidad comprenda sobre qué se están tomando las decisiones" (Sofield 2003 en Cole 2006, p.632). De hecho, como señala Ashley et al. (2001), los pobres tienen una débil comprensión sobre los turistas así como sobre la forma en que funciona la industria turística, así que el cambio de esta situación sería muy importante para tomar decisiones adecuadas e informadas en relación a su propio desarrollo turístico. Schilcher (2007) refuerza esta idea argumentando que más que promover el turismo (en cualquiera de sus formas) como una cura a la pobreza, los tomadores de decisiones, donadores e investigadores deberían (re-)descubrir el conocimiento local en lugar de solo utilizar la retórica bajo la sombrilla del desarrollo alternativo o sustentable. Nadkarni (2008, p.459) agrega que debería implementarse un mecanismo específico en "creación, retención, intercambio, devolución, interpretación y tratamiento del conocimiento" (que denomina como K-CREDIT) en turismo en el nivel de la base social, lo cual impulsaría económicamente destinos en desventaja, con la premisa de que el déficit de conocimiento perpetúa un círculo vicioso de carencias económicas y pobreza.

La importancia de compartir y transferir conocimiento como una forma de derribar barreras que impiden la interacción, la comunicación saludable y la colaboración está siendo considerada como un fuerte elemento en los recientes discursos de sostenibilidad. Esto es además apuntalado por el concepto de knowledge brokerage (Sheate and Partidário, 2010), que es más amplio que la mera transferencia de conocimiento ya que incluye también elementos como la comunicación, interacción, contribución al entendimiento mutuo, así como la acción efectiva y eficiente.

La Comunicación es entonces una dimensión central para la construcción de capacidades en tanto que es una condición para compartir conocimiento que a su vez conduce al empoderamiento. De hecho, la comunicación constituye la base de todo el desarrollo humano, en cualquier contexto (Romanow and Bruce, 2006). Las teorías actuales de la comunicación para el desarrollo consideran que la falta de poder político, económico y cultural de los sectores en los niveles más bajos es el principal problema a solucionar en el desarrollo (White, 2004). La mayoría de la población de países en desarrollo no tiene acceso a la educación, ayuda técnica, buena salud y vivienda necesarias para hacer una contribución al desarrollo. De ahí que se requiera que tenga lugar la construcción de capacidades en el contexto de las prácticas y procesos de comunicación. Lo más importante que pueden hacer las comunidades para construir capacidades es implicarse en un diálogo multidireccional con todos los actores involucrados con la comunidad (Romanow and Bruce, 2006).

Para focalizar el envolvimiento de los actores es crucial generar ventajas estratégicas competitivas a través de redes (Cooper, 2006) que, asociadas a agrupamientos de intereses, son precondiciones para la innovación y la construcción de capacidades de la comunidad (Dredge, 2006). Aún así, muchas organizaciones y actores necesitan ser persuadidos de que la cooperación fortalecerá su propia competitividad, y existe entonces una necesidad de demostrar los beneficios de la cooperación en el destino (Cooper, 2006; Dredge, 2006; Shaw \& Williams, 2009). Las teorías de trabajo en redes y clusters están siendo utilizadas cada vez más para explicar el papel del turismo en el crecimiento local y estimular el desarrollo regional tal como lo presentan Novelli et al. (2006).

La Gobernanza es también un elemento crítico para lograr el desarrollo sostenible. Su concepto es complejo y existe una gran variedad en la forma en que las agencias de desarrollo, las organizaciones internacionales y las instituciones académicas consideran su significado. La definición del UNDP (2000, p.1) de gobernanza democrática se enfoca en el papel esencial que ésta desempeña en los ODMs, considerándola como "el sistema de valores, políticas e instituciones mediante las cuales una sociedad administra sus asuntos económicos, políticos y sociales a través de interacciones dentro y entre el sector público, la sociedad civil y el sector privado". En lo que se refiere a la estructura de la gobernanza en turismo, hay que con- 
siderar diversos actores, que impactan el desempeño de la sostenibilidad del sector: autoridades públicas en todos los niveles de gobierno, agentes del sector privado nacional y extranjero, turistas y comunidades locales (Dinica 2009) y la sociedad civil.

Aunque se considera que los determinantes aquí presentados son cruciales para que una intervención para el desarrollo en turismo sea exitosa, ello no significa que sean los únicos. Sin embargo, los autores consideran que si los programas de cooperación logran tener una influencia positiva en todos, o al menos en alguno de los determinantes, es entonces posible producir un efecto positivo global a largo plazo para el desarrollo turístico del destino.

\section{El Programa UNWTO.Volunteers de la Fundación UNWTO. Themis}

La Organización Mundial del Turismo desempeña un papel central y decisivo en la promoción del desarrollo de un turismo responsable, sostenible y accesible para todos, prestando especial atención a los intereses de los países en desarrollo. Desde 2003, es un organismo especializado de las Naciones Unidas y representa la principal institución internacional en el campo del turismo. Constituye un foro mundial para debatir cuestiones de política turística y una fuente útil de conocimientos especializados en este campo. $\square$ Entre sus miembros figuran 154 países, siete territorios y más de 400 Miembros Afiliados que representan al sector privado, a instituciones de enseñanza, a asociaciones de turismo y a autoridades turísticas locales (UNWTO, 2009).

En 2005, la OMT inició el Programa UNWTO.TedQual Volunteers a través de la Fundación UNWTO.Themis, con la misión de: "Contribuir a los Objetivos de Desarrollo del Milenio de las Naciones Unidas promoviendo al turismo como instrumento de desarrollo a través de la educación y formación de profesionales voluntarios para apoyar a los Estados Miembros de la OMT, difundiendo el espíritu del voluntariado" (UNWTO.Themis, 2008).

Los Proyectos en el ámbito del Programa UNWTO.Volunteers se realizan en un país de acogida, cuya Administración Nacional de Turismo ha presentado un proyecto de desarrollo, e involucra a expertos en turismo y jóvenes voluntarios especializados en turismo y cooperación para el desarrollo para llevar a cabo un proyecto de 6 meses, incluyendo 3 a 4 semanas de trabajo de campo en el país de acogida (UNWTO.Themis, 2008).

El principio básico es aprovechar la experiencia del país solicitante, de los expertos de la OMT y del personal de la OMT, y brindar asistencia al país anfitrión en la identificación de productos turísticos de gran potencial y en el desarrollo de alternativas de posicionamiento estratégico que contribuyan al desarrollo sostenible del turismo como alternativa económica, buscando la generación de beneficios socioeconómicos. En este planteamiento juega un papel fundamental la gobernanza en el turismo, de manera que, la participación activa del sector público, privado y de la sociedad civil en todas las fases del proce- so propicia el desarrollo turístico y el equilibrio medioambiental.

El Programa UNWTO.Volunteers tiene los siguientes objetivos:

- Apoyar a los Estados Miembros de la OMT en la formulación e implementación de planes, programas y proyectos a través de la contribución técnica de expertos y voluntarios de la OMT;

- Capacitar de una manera teórico-práctica a profesionales con la adecuada vocación y aptitudes en el campo del turismo como instrumento para el desarrollo: Voluntarios;

- Difundir, a través de la educación y la formación, las políticas de la OMT en el campo del turismo, especialmente, la condición del turismo como instrumento de desarrollo y su potencialidad para contribuir en la reducción de la pobreza;

- Difundir los ideales de servicio y solidaridad arraigados en la esencia del voluntariado.

\section{Metodología}

El estudio de caso fue el método utilizado que incidió en el proyecto "Chiapas 2015: Plan de Estrategia y Competitividad Turística” del Programa UNWTO.Volunteers. Se utilizaron las técnicas de recolección de datos secundarios por medio de la investigación documental y de datos primarios a través del trabajo de campo, con entrevistas semi-estructuradas y a profundidad, complementadas con la observación participante de los autores, aunque con diferentes papeles.

La investigación documental consideró varias fuentes escritas, siguiendo un proceso de localización, selección y clasificación de la información, en diversas fases del proyecto. Por otra parte, el trabajo de campo complementó la investigación documental, sirviendo para sustentar los resultados y la propuesta planteada.

Dicho proyecto se logró desarrollar con la participación de once voluntarios internacionales del Programa UNWTO.Volunteers provenientes de Portugal, España, Brasil, Guatemala y México, voluntarios egresados del "Curso Universitario Turismo y Cooperación Internacional para el Desarrollo", en 2007 y 2008, de la OMT. Asimismo, participaron dos expertos técnicos en turismo de nacionalidad mexicana, quienes coordinaron los trabajos de campo y la redacción del documento final.

Los voluntarios y expertos iniciaron las primeras exploraciones en el mes de agosto de 2008 y concluyeron con el trabajo de campo en el mes de octubre de 2008. Los trabajos que se desarrollaron en la parte de exploración se realizaron por equipos integrados en forma interdisciplinaria donde se presentaron los primeros avances en la concentración en el área de estudio.

El trabajo de campo consistió en la visita técnica en los cinco municipios con el mismo equipo de trabajo para evaluar la calidad estética de los atractivos turísticos naturales y socioculturales, los recursos turísticos, la infraestructura, la estructura y la superestructura turísti- 
ca. Para la obtención de la información se visitaron los diferentes atractivos, se realizaron entrevistas con prestadores de servicios turísticos, funcionarios, turistas y la población local. Además fue realizado un análisis DAFO para identificar las debilidades, amenazas, fortalezas y oportunidades en cada municipio, a través de un taller de planeación estratégica con los actores mencionados para cada municipio.

\section{El proyecto "Chiapas 2015: Plan de Estrategia y Competitividad Turística"}

\section{Marco geográfico}

Chiapas, uno de los cinco estados de la República Mexicana que forma parte del Mundo Maya, cuenta con una gran riqueza en arqueología, cultura y naturaleza, concentrándose en un espacio territorial de 75634 km2 (Figura 1). Representa el 3,8\% de la superficie del país, colinda al norte con Tabasco; al este con la República de Guatemala, al sur con el Océano Pacífico y al oeste con los estados de Oaxaca y Veracruz. En el estado se concentra el $30 \%$ del agua superficial del país, producto de los ríos Grijalva, Usumacinta, Lacantún, Jataté y Suchiate; las presas: Belisario Domínguez, Chicoasén, Nezahualcóyotl y Peñitas; y de los Lagos de Montebello, Colón y Miramar.

Existen 37 áreas naturales protegidas, entre las que destacan Montes Azules, La Sepultura, La Encrucijada, El Ocote, y El Triunfo. En el territorio chiapaneco existen 19386 localidades, distribuidas en 118 municipios, agrupados en 9 regiones económicas.

Las atracciones turísticas más importantes de la región incluyen restos arqueológicos de la civilización maya y una diversidad de prácticas culturales de diferentes grupos de origen maya, entre ellos chamulas, tzotziles y tzeltales, que residen en medio de una exuberancia natural. A partir de la relación entre la riqueza étnica y natural, el desarrollo económico regional ha incorporado el desarrollo del turismo cultural y arqueológico, y el ecoturismo y de aventura (Coronado, 2008).

De esta manera, Chiapas opta por desarrollar un turismo responsable y solidario por la calidad estética de los atractivos naturales y culturales que tiene este estado del sureste mexicano, que son únicos en el país. Se pueden mencionar como a categoría de ejemplos los siguientes lugares: Cascadas de Agua Azul, Río Usumacinta, Lago Miramar en el corazón de la Selva Lacandona, Lagos de Montebello, la Cascada el Chiflón. Asimismo, Chiapas concentra un alto porcentaje de especies de fauna, en específico aves. En cuanto a la arqueología, se destacan los ejemplos de centros ceremoniales de la cultura Maya como Bonampak, Yaxchilán, Palenque, Toniná, Chinkultik y otros sitios, la mayoría en las zonas rurales.

El turismo es visto como una oportunidad para el desarrollo económico de la región y como prioritario en los planes gubernamentales en el combate a la marginalización de los Pueblos Indios (Coronado, 2008). Asimismo, el desarrollo turístico resulta de múltiples planes gubernamentales y la industria turística ha evolucionado a partir de diversas iniciativas tanto individuales como colectivas, públicas o privadas.

\section{Caracterización del Proyeto "Chiapas 2015"}

El Proyecto "Chiapas 2015" inicia con la solicitud del gobierno del Ayuntamiento de Tuxtla Gutiérrez, capital del Estado de Chiapas, ante las autoridades estatales y nacionales correspondientes, quienes, a su vez, presentaron la petición a la OMT. A esta solicitud se sumaron las de los municipios de Chiapa de Corzo, Comitán de Domínguez, San Cristóbal de las Casas y Palenque, también del Estado de Chiapas.

Con la finalidad de concretar los alcances del Proyecto, en el mes de octubre de 2007 se llevó a cabo una misión exploratoria en la que participaron el Director Ejecutivo de la Fundación UNWTO.Themis, Vicente J. Molés y la consultora Cecilia García, como expertos de la OMT, quienes acompañados por las autoridades turísticas visitaron las ciudades de Tuxtla Gutiérrez, Chiapa de Corzo, San Cristóbal de las Casas, Comitán de Domínguez y Palenque, entrevistando autoridades estatales, municipales y representantes del sector privado.

El Convenio para la elaboración del Proyecto "Chiapas 2015", fue firmado con fecha 8 de agosto de 2008, participando en este documento las autoridades nacionales, estatales y municipales, así como la contraparte de la Organización Mundial del Turismo, formalizando de esta manera la ejecución del proyecto.

Derivado de los trabajos realizados y siguiendo el enfoque metodológico adoptado por el grupo de trabajo, se tomó la decisión de que el Proyecto Chiapas 2015 estuviera conformado por los siguientes elementos:

1. Diagnóstico de la situación actual del Sistema Turístico de los Destinos Seleccionados, para identificar y evaluar los diversos elementos que constituyen el sistema, así como los elementos externos y el análisis estratégico de los destinos, con base en los intereses y deseos de los protagonistas de la actividad turística en dichas localidades, como requisito y condición previa a la formulación de propuestas de desarrollo.

2. Propuesta de Modelo de Desarrollo, que plantea los criterios generales para un desarrollo turístico sostenible y establece los componentes que configuran el modelo:

- Estructuración turística del espacio;

- Determinación de objetivos y visión del desarrollo turístico para la zona motivo de estudio;

- Propuesta de posicionamiento turístico para el área de estudio, con base a las oportunidades que presenta el entorno de mercado y a las posibilidades objetivas de las que disponen cada uno de los destinos incluidos en el Proyecto;

- Evaluación de diferentes opciones de desarrollo y estrategia para el área motivo del estudio;

- Elección de una propuesta estratégica, es decir, selección de una cartera de negocios/productos prioritarios para cada centro turístico identificado y para el conjunto del área de estudio, la priorización de públicos/segmentos por productos y centros, y la priorización de mercados 


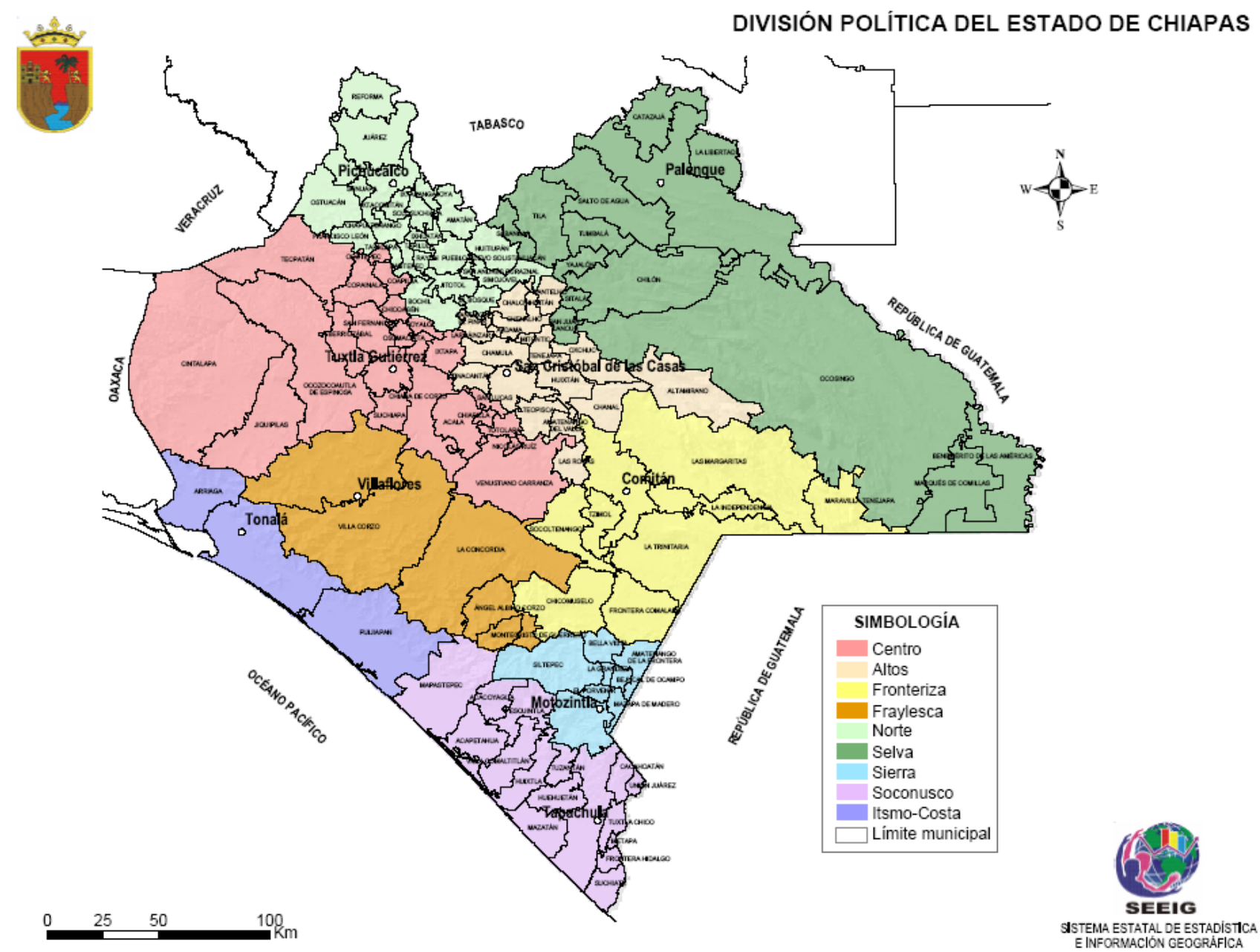

Figura 1.- División Política y Regiones Económicas del Estado de Chiapas. Fuente: Sistema Estatal de Estadística e Información Geográfica, Gobierno del Estado de Chiapas (2008)

geográficos por productos y centros.

La identificación y priorización de los campos de acción es el resultado, por una parte, de las propias necesidades y requerimientos que plantea el Modelo de Desarrollo y, por la otra, de la valoración que han realizado los propios protagonistas de la actividad turística de los destinos participantes tanto en entrevistas hechas a informantes clave en cada uno de los destinos, como a través de los talleres sectoriales celebrados. Estos talleres fueron concebidos y organizados desde dos perspectivas: a) Desde una perspectiva territorial, para recoger los intereses, inquietudes, opiniones y planteamientos de todos los agentes que desarrollan su actividad turística en un mismo espacio físico, económico, social, administrativo, etc. y b) Desde una perspectiva sectorial, para profundizar determinados aspectos que afectan a actividades y colectivos específicos como hoteleros, operadores, administración municipal, etc.

3. Programas específicos de actuación, que identifican y priorizan los ámbitos de actuación para crear, mejorar y reforzar las condiciones que permitan la implementación del Proyecto Chiapas 2015.

Una vez definido el modelo de desarrollo e identificados los campos de acción prioritarios, la siguiente fase del proyecto consistió en la elaboración de programas de actuación detallados para trabajar de manera sistemática en mejorar las condiciones generales y específicas de los centros y productos turísticos.

Dichos programas de actuación se concretaran en cinco propuestas, que abarcan los principales temas identificados como parte del diagnóstico, como son:

1. Coordinación interinstitucional - formación de un equipo de trabajo con la integración de los dos niveles de gobierno (estatal y municipal) con la iniciativa privada (hoteleros, gerentes de restaurantes, agentes de viajes, guías de turistas, transportadores) y la sociedad civil.

2. Capacitación turística - formación de recursos huma- 
nos especializados en la administración y gestión de empresas turísticas, atención a los visitantes, fomento de cultura turística, que contribuya a la prestación de servicios con calidad.

3. Creación y consolidación de productos turísticos - diseño de nuevos productos que permitan diversificar y complementar la oferta turística existente en cada destino integrándose de manera coordinada con los cinco municipios involucrados en el proyecto. Con ello, se pretende crear un corredor turístico funcional organizado de manera sistemática con todos los actores involucrados, y además contribuir a la consolidación de productos turísticos ya existentes en la región estudiada.

4. Plan de marketing - elaboración de un plan de marketing como marco de referencia para los cinco municipios involucrados, basado en el potencial turístico evaluado por el equipo de la OMT en conjunto con los actores locales. Dicho plan debe contemplar un estudio de mercado (segmentación de mercado de acuerdo con el posicionamiento de cada producto, estrategias de comunicación), que coadyuve a la consolidación del corredor turístico.

5. Para el desarrollo adecuado de las propuestas fue considerado como condiciones previas esenciales al éxito del programa el mejoramiento de las infraestructuras y equipamientos, mejoramiento de la imagen urbana, y atención a problemas sociales y ambientales como son ejemplos la venta ambulante, manejo de basura, entre otros.

La participación de la OMT en Chiapas durante el trabajo de campo fue considerado relevante por los distintos actores locales, en específico porque se generó una expectativa muy alta en cuanto a la perspectiva de ser detonante en términos de planificación estratégica del turismo con una participación más amplia a nivel de los municipios y de actores locales involucrados.

\section{Reflexiones finales y conclusiones}

Con frecuencia se cree que la variedad y calidad de los atractivos en un destino y su zona de influencia son los componentes determinantes para establecer la competitividad de un destino. El trabajo de campo realizado en Chiapas por el grupo de voluntarios confirmó este postulado. Esta idea bastante extendida parece haber motivado iniciativas para entrar al mercado turístico, o bien para despertar, entre las comunidades, el sector empresarial y el sector público local, expectativas desmedidas en torno a los beneficios que puede generar el turismo, en la creencia de que basta contar con paisajes y atractivos para asegurarse una posición en el mercado.

Entre los diferentes actores involucrados en la actividad turística de los municipios que fueron receptores del Programa UNWTO.Volunteers, encontramos que se dejaba de lado, o no se conocían elementos indispensables para consolidar un destino turístico, tales como: la condición de los factores productivos y de las industrias de apo- yo; la ubicación geográfica; el clima de negocios; los costos de transportación; la información sobre los mercados; la disponibilidad y especialización de los recursos humanos; así como las habilidades existentes para la planeación, promoción y comercialización.

Consideramos que un valor fundamental del Programa UNWTO.Volunteers es que pone especial énfasis en el desarrollo de capacidades y habilidades intelectuales en los destinos turísticos como piedra angular que orienta el trabajo de las instituciones, impulsa a las empresas e integra a los diferentes actores hacia un propósito compartido de largo plazo, a partir del reconocimiento de la complejidad de la actividad turística. Esta complejidad obedece no sólo al conjunto de elementos que convergen a estructurarla al interior del sistema, sino a las múltiples relaciones que mantiene el sistema turístico con el entorno político, social, económico, ambiental y tecnológico. Se destaca el conocimiento como recurso estratégico para la generación de riqueza a través de la identificación de oportunidades. Bajo esta perspectiva, el conocimiento puede, incluso, crear atractivos turísticos allí en donde antes no los había.

$\mathrm{Al}$ fomentar la representatividad y considerar los intereses e iniciativas referentes al turismo en sentido amplio de la sociedad civil y del sector privado: empresas, sindicatos, instituciones del conocimiento (centros de investigación, universidades, otras instituciones de enseñanza, etc.), organizaciones no gubernamentales, grupos ciudadanos, a través de la investigación previa, entrevistas personales y de los talleres realizados durante el trabajo de campo, el Programa UNWTO.Volunteers comunica al destino receptor del programa, que debido a la existencia de estas estructuras y sus relaciones dinámicas, que finalmente son las que explican la aparición y el desarrollo del turismo, el impulso a la actividad no puede reducirse a elementos simples como la existencia de atractivos. Se advierte también que las condiciones y estructura de las empresas y las industrias resultan básicas para la competitividad de un destino. Por lo tanto, los esfuerzos para mejorarla deben enfocarse hacia ellas, aunque no únicamente, en tanto que las administraciones públicas locales también tienen un papel relevante en cualquier proceso de desarrollo turístico y de mejora de la competitividad.

$\mathrm{Al}$ trabajar con equipos interdisciplinarios, el Programa UNWTO.Volunteers enfatiza que el proceso de desarrollo turístico es amplio por naturaleza, ya que engloba programas económicos, tecnológicos, ambientales, sociales, culturales y políticos, poniendo de relieve las interrelaciones entre las distintas esferas.

Consideramos que a través de la propuesta de Programas de Actuación específicos, el Programa UNWTO. Volunteers en el caso del "Proyecto Chiapas 2015", si le fuera dado el debido seguimiento, podría apoyar la labor de las autoridades federales, estatales y municipales solicitantes, así como de las empresas y sociedad civil para priorizar, enfocar y posicionar al destino, asegurándose de que se cumplirían las condiciones para la sostenibilidad, ayudando a satisfacer las expectativas de los actores in- 
volucrados y presentando opciones para la utilización de los recursos públicos y privados que apuntasen a la rentabilidad económica, social y ambiental a largo plazo, y que acabarían por contribuir para la atenuación de la pobreza.

Sin embargo, con base en conclusiones preliminares de un estudio independiente realizado en 2011 por los autores, se considera que en general el programa no ha correspondido a las elevadas expectativas generadas por varias razones: 1) Los actores que participaron en la fase del trabajo de campo no fueron informados de las conclusiones del estudio y de su posible seguimiento, verificándose falta de comunicación por parte de las autoridades, 2) las mismas autoridades locales han dado seguimiento muy parcial o nulo, esencialmente por los cambios de periodos y actores políticos, 3) en algunos municipios se observó que la iniciativa privada y la sociedad civil manifiestan apatía en una participación más activa, 4) algunos factores externos pueden haber influenciado en el proceso de implementación (ejemplos: crisis económica financiera internacional desde finales de 2008, influenza en 2009, etc.).

Podemos así concluir, con el ejemplo del "Proyecto Chiapas 2015", que el Programa UNWTO.Volunteers presenta características en su diseño y implementación que nos hacen creer que los resultados futuros podrán tener un impacto positivo en los destinos objeto de intervención, ya que se preconiza e implementa una efectiva participación de todos los actores locales, del sector público, privado y de la sociedad civil, en la discusión de los Planes de Desarrollo Estratégico contribuyendo, a través de la transferencia de conocimiento y de una comunicación más reforzada, ante todo para el refuerzo de la Gobernanza en el destino. De hecho, el programa presenta un fuerte potencial para poder constituirse como un instrumento eficaz para detonar procesos de desarrollo turístico basado en una planificación estratégica y participativa, considerando en sus principios básicos los determinantes analizados en el marco teórico de este articulo, al enfatizarse la importancia de la participación de diferentes actores locales en el proceso de planificación estratégica del turismo, del reparto de información y del proceso de "knowledge brokerage" a través de una comunicación fluida entre todos los niveles, la planificación integrada abarcando clusters turísticos. No obstante, lo que se verificó con el estudio de caso de Chiapas es que terminada la intervención de la OMT y después de presentado el informe final a las autoridades estatales, eses factores considerados determinantes al inicio no fueron efectivamente llevados en consideración. Siendo una de las causas para la falta de seguimiento del programa apuntadas por los actores locales los cambios políticos y de los responsables de las autoridades locales, se considera que una condición esencial para dar seguimiento en su implementación seria la creación de un comité o consejo de turismo local integrado por un equipo amplio de actores del sector público, privado y social civil, cuyo liderazgo no dependiera del gobierno de turno para evitar la discontinuidad del programa.

Para poder analizar en profundidad los impactos que este tipo de programas pueden generar en los destinos se considera determinante llevar a cabo monitorizaciones y evaluaciones, que involucren las comunidades beneficiadas, para determinar el impacto de los proyectos una vez que se hacen operativos (Bamberger, 2000), e introducir los cambios necesarios a la obtención de mejores resultados en programas futuros de cooperación internacional para el desarrollo a través del turismo.

\section{Agradecimientos}

Los autores agradecen el inestimable apoyo dado en la fase de trabajo de campo llevada a cabo en Julio y Agosto de 2011 en Chiapas a las siguientes autoridades: Rector de la Universidad Intercultural de Chiapas, Doctor Andrés Fábregas Puig; Director Regional del Estado de Tabasco del Instituto Nacional de Arqueología e Historia (INAH) y del sitio Arqueológico de Palenque en Chiapas, Doctor Juan Antonio Ferrer; Rector de la Universidad Intercultural de Tabasco, Ing. Pedro Pérez Luciano.

\section{Bibliografía}

Ashley, C., Roe, D. and Goodwin, H.

2001 Pro-poor Tourism Strategies: Making Tourism Work for the Poor - A review of experience. London: Overseas Development Institute.

Bamberger, $\mathrm{M}$.

2000 "The Evaluation of International Development Programs: A View from the Front". American Journal of Evaluation, 21 (1): 95-102.

Blake, A., Arbache, J., Sinclair, M.T. and Teles, V.

2008 "Tourism and Poverty Relief". Annals of Tourism Research 35(1): 107-26.

Brohman, J.

1996 "New directions in tourism for third world development". Annals of Tourism Research, 23 (1): 48-70.

Carbone, M.

2007 "Sustainable tourism in developing countries: poverty alleviation, participatory planning and ethical issues". The European Journal of Development Research, 17(3): 559-565.

Chok, S., Macbeth, J. and Warren, C.

2007 "Tourism as a tool for poverty alleviation: a critical analysis of pro-poor tourism and implications for sustainability". Current Issues in Tourism, 10(2/3): 144-65.

Cole, S.

2006 "Information and Empowerment: The Keys to Achieving Sustainable Tourism". Journal of Sustainable Tourism, 14(6): 629-44.

Cooper, C.

2006 "Knowledge Management and Tourism". Annals of Tourism Research, 33 (1): 47-64.

Coronado, G.

2008 "Insurgencia y turismo: reflexiones sobre el impacto del turista politizado en Chiapas". Pasos Revista de 
Turismo y Patrimonio Cultural, 6(1): 53-68.

Dinica, $\mathrm{V}$.

2009 "Governance for sustainable tourism: a comparison of international and Dutch visions". Journal of Sustainable Tourism, 17 (5): 583-603.

Dredge, D.

2006 "Policy networks and the local organisation of tourism". Tourism Management, 27: 269-280.

Dubois, A.

2007 "Fundamentos teóricos para el desarrollo humano local: las capacidades colectivas". Ponencia presentada al Congreso El desafío del desarrollo humano. Propuestas locales para otra globalización, Hegoa/EPVEHU, Bilbao, febrero 2007. URL: www.hegoa.ehu.es/ congreso/bilbo/cas/2_comu (consultado en 10-06-2011)

Eade, D.

1997 Capacity-Building: An Approach to People-Centred Development. Oxford UK and Ireland: Oxfam.

Fayos-Solà, E.

2004 "Política turística en la era de la globalización". En Martín, J. A. (Coord.), Mediterráneo económico - Las nuevas formas del turismo, 5, 215-232. URL: http:// dialnet.unirioja.es/servlet/articulo?codigo $=2158794$ (consultado en 20-10-2009)

Fayos-Solà, E. \& Pedro, A.

2001 "Globalization, National Tourism Policies and International Organizations". En Cooper, C. \& Wahab, S. (Eds), Tourism in the Age of Globalization (45-65). London: Routledge.

Ferguson, L.

2007 "The United Nations World Tourism Organisation". New Political Economy, 12(4): 557 - 568.

Gobierno del Estado de Chiapas

2008 "Sistema Estatal de Estadística e Información Geográfica". URL: http://www.inegi.org.mx/snieg/ (consultado en 20-07-2009)

Hawkins, D. E. \& Mann, S.

2007 "The World Bank's Role in Tourism Development". Annals of Tourism Research, 34(2): 348-363.

Lima, S., Eusébio, C. \& Partidário, M.R.

2010 "Tourism and poverty alleviation: the role of the international development assistance". En Sarmento, M. \& Matias, A. (Eds), Economics and Management of Tourism: Tendencies and Recent Developments. Lisboa: Universidade Lusíada Editora, Colecção Manuais [in press].

Nadkarni, S.

2008 "Knowledge Creation, Retention, Exchange, Devolution, Interpretation and Treatment (K-CREDIT) as an Economic Growth Driver in Pro-Poor Tourism". $\mathrm{Cu}$ rrent Issues in Tourism, 11(5): 456-72.

Novelli, M., Schmitz, B. and Spencer, T.

2006 "Networks, clusters and innovation in tourism: a UK experience". Tourism Management, 27: 1141-152.

Partidário, M. R. \& Wilson, L.

2009 "Institutional and Professional Capacity-Building for SEA". En Sadler, B., Aschmann, R., Dusik, J., Fis- cher, T., Partidário, M.R. \& Verheem, R. (Eds), Handbook on Strategic Environmental Assessment. London: Earthscan.

Romanow, P. and Bruce, D.

2006 "Communication and Capacity Building: Exploring Clues from the Literature for Rural Community Development”. Journal of Rural and Community Development, 1: 131-54.

Shaw, G. and Williams, A.

2009 "Knowledge transfer and management in tourism organisations: an emerging research agenda". Tourism Management, 30: 325-35.

Sheate W. R. and Partidário M. R.

2010 "Strategic approaches and assessment techniquesPotential for knowledge brokerage towards sustainability". Environmental Impact Assessment Review, 30: 278-88.

Scheyvens, R.

1999 "Ecotourism and the empowerment of local communities". Tourism Management, 20: 245-49.

Scheyvens, R.

2007 "Exploring the Tourism-Poverty Nexus". Current Issues in Tourism, 10(2,3): 231-254.

Scheyvens, R. \& Momsen, J. H.

2008 "Tourism and poverty reduction: issues for Small Island States". Tourism Geographies, 10(1): 22-41. Schilcher, D.

2007 "Growth versus equity: the continuum of pro-poor tourism and neoliberal governance". Current Issues in Tourism, 10 (2/3): 166-92.

Tosun, C.

2001 "Challenges of sustainable tourism development in the developing world: the case of Turkey". Tourism Management, 22: 289-303.

Tosun, C. and Timothy, D.

2003 "Arguments for community participation in tourism Development”. Journal of Tourism Studies, 14 (2): $2-11$.

UN - United Nations

2006 The Millennium Development Goals Report 2000. New York: UN.

UN - United Nations

2009 "Objetivos de Desarrollo del Milenio 2015". URL: http://www.un.org/spanish/millenniumgoals/ (consultado en 20-07-2009)

UNDP - United Nations Development Programme

1997 Human Development Report 1997. New York: Oxford University Press.

2000 "Good Governance: a User's Guide", http://gaportal. org/sites/default/files/undp_users_guide_online_version.pdf

UNWTO - United Nations World Tourism Organization 2001 Tourism in the Least Developed Countries. Madrid: WTO.

2004 Tourism and Poverty Alleviation Recommendations for Action. Madrid: WTO.

2008 "Tourism Highlights - 2008 Edition". URL: http:// 
www.unwto.org/facts/eng/highlights.htm (consultado en 20-02-2009)

2009 "Cooperation Activities". URL: http://ekm.unwto. org/english/activities.php (consultado en 10-03-2009)

UNWTO.Themis

2008 "Misión". URL: http://www.unwto-themis.org/en/ who_we_are/mission (consultado en 10-02-2008)

White, R.

2004 'Is 'Empowerment' The Answer? Current Theory and Research on Development Communication". Gazette: The International Journal for Communication Studies, 66 (1): 7-24.

Zhao, W. \& Ritchie, J. R. B.

2007 "Tourism and poverty alleviation: an integrative research framework". Current Issues in Tourism, 10(2): 119-143.

Recibido:

Reenviado:

Aceptado:

$02 / 12 / 2010$

$14 / 09 / 2011$

$17 / 10 / 2011$ 